\title{
Blazar variability and gamma-ray emission - signatures for leptonic and hadronic jet gamma-ray production models
}

\author{
Richard J. Britto (on behalf of the Fermi-LAT Collaboration)* \\ Department of Physics, University of the Free State, PO Box 339, Bloemfontein 9300, South \\ Africa \\ E-mail: brittor@ufs.ac.za, dr.richard.britto@gmail.com
}

\section{Johannes P. Marais, Pieter J. Meintjes, Brian van Soelen}

Department of Physics, University of the Free State, PO Box 339, Bloemfontein 9300, South Africa

\begin{abstract}
Blazars are known to be the most energetic sources (apart from gamma-ray bursts) we can observe in the Universe. They constitute the brightest type of radio-loud active galactic nuclei whose Doppler boosted relativistic jets are close to our line of sight. Blazars release a tremendous amount of non-thermal radiation throughout the whole electromagnetic spectrum, and often exhibit outbursts. These outbursts generally last between a few days and several months. The fast and frequent variability characteristics of many of these sources make possible detailed multiwavelength time-domain analysis. Also, we study the evolution of their spectral features in order to constrain physical mechanisms that drive the radiation production within the jets. After introducing the most common methods used to discriminate between leptonic and hadronic scenarios, we report studies on the flat spectrum radio quasar 3C 454.3 (one of the brightest blazars in gamma rays) to show how time-domain and spectral analysis can provide constraints on the size and the location of the gamma-ray emitting region. We also present our work with optical data obtained from South Africa based telescopes to carry out multiwavelength studies of blazars.
\end{abstract}

Frontier Research in Astrophysics - II

23-28 May 2016

Mondello (Palermo), Italy

\footnotetext{
* Speaker.
} 


\section{Introduction}

The nature of Quasi-stellar radio sources, most commonly known as quasars, was a puzzle for astronomers and astrophysics for many years, and particularly since the time their redshifts were measured in the early 1960s [1, 2, 3, 4]. These sources are currently understood within the framework of the unified model of active galactic nuclei (AGNs) [5], where a supermassive black hole $(\mathrm{SMBH})$ of $10^{6}-10^{9}$ solar masses lies at the centre of the AGN, surrounded by a disk of accreted material heated by frictions generated by the strong gravitation field of the SMBH, and falling onto the SMBH by spiralling. This generates thermal radiation, mainly in the ultraviolet. If a quasar is radio-loud, a pair of twin jets of relativistic plasma originates from within the sub-parsec scales from the SMBH, and perpendicular to the accretion disk. These jets are believed to be due to a spinning SMBH in a strong magnetic field. Particles interact through internal and external processes within the jets, and generate radiation that spreads over the whole electromagnetic spectrum, from radio waves to gamma rays. Due to the relativistic beaming of the radiation within the narrow jets, when the Earth is close to the direction of the jet, the source appears extremely bright, and is then called a "blazar". The non-thermal radiation emitted within the jet then dominates the signal received from the source.

Blazars are classified into two categories: flat spectrum radio quasars (FSRQs), and BL Lacs. FSRQs are usually more luminous, found at higher redshifts, and surrounded by relatively dense radiation fields - called the broad-line region (BLR) - at the parsec scale from the SMBH. Their ultraviolet and optical spectra are characterised by broad line features, whereas in the same wavelength range, BL Lacs' spectra are quasi-featureless, or have very narrow optical lines (equivalent width $<5 \AA$ in their rest frame). Several interesting reviews on blazars and the current status of these studies were presented by e.g. $[6,7,8]$.

The spectral energy distribution of blazars is characterised by a double hump feature. The peak of the low energy (LE) hump is used to define the blazar as a Low, Intermediate or High Synchrotron Peak blazar (LSP, ISP or HSP, respectively - see Table 1). Most of the FSRQs are LSP blazars. BL Lacs that belong to the LSP, ISP or HSP blazar class are called LBL, IBL or HBLs. The low energy peak, spreading from radio to somewhere between optical and X-rays, is understood to be due to synchrotron radiation produced in the jets by relativistic electrons. This is well established and supported by polarisation measurements $[9,10,11,12]$. The second hump spreads from $\mathrm{X}$-rays to $\mathrm{GeV}$ or $\mathrm{TeV}$ gamma rays, and can be due to both leptonic and hadronic processes.

Blazars exhibit dramatic variability patterns during flaring activities. The spectral energy distribution (SED) of a particular source can be dramatically different for separate flaring episodes, both in intensity and spectral shape. This could suggest that different flaring phases could be interpreted by different mechanisms (leptonic or hadronic), or simply that the detected radiation originates from different regions - usually called blobs (compact region often considered as spherical) or knots - within the jets (Figure 1). This dramatic variability that we observe in blazars constitute a wealth of information that enables us to investigate the physical mechanisms occurring in blazars through appropriate modelling of these objects.

A particularly well-suited instrument to study such variability patterns is the Fermi GammaRay Space Telescope. Launched on 11 June 2008, this space telescope is orbiting the Earth every 
Table 1: Peak positions of the blazar sequence.

\begin{tabular}{c|c}
\hline Blazar SED class & Synchrotron Peak position $v_{\text {peak }}^{s}(\mathrm{~Hz})$ \\
\hline LSP & $v_{\text {peak }}^{s}<10^{14}$ \\
ISP & $10^{14}<v_{\text {peak }}^{s}<10^{15}$ \\
HSP & $v_{\text {peak }}^{s}>10^{15}$ \\
\hline
\end{tabular}

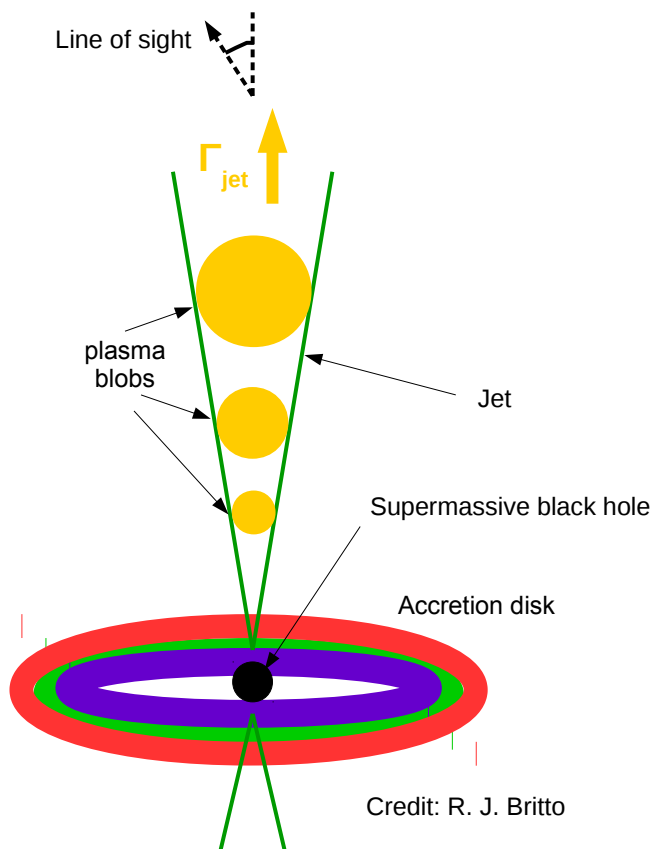

Figure 1: Cartoon of gamma-ray emitting plasma blobs that may be at certain distances from the SMBH. Though it is often assumed that a blob is spherical and that its diameter is a section of the conical jet, these hypotheses are still debated, and may be correct only for some specific flaring events. Also, the presence of a BLR - as for FSRQs - may be at the origin of gamma-ray absorption whenever the blob is located below it.

$1.5 \mathrm{hr}$. Its main instrument, the Large Area Telescope (LAT) sees the whole sky every two orbits [13], thanks to a broad field of view of 2.4 sr. It is sensitive to photons in the $20 \mathrm{MeV}$ to $>300$ $\mathrm{GeV}$ range. Since Fermi operates in the survey mode for most of its observation time, the LAT is an ideal instrument for variability studies of bright sources in sub-day time scales, for any period of time since 4 August 2008, date of the earliest public science data set.

In this paper we discuss some of the possible constraints that can be given through time-domain analysis and spectral studies to investigate emission in blazars. In section 2, we review some basic concepts related to the leptonic versus hadronic challenges of identifying the types of radiation emission mechanisms. In section 3, we present some recent work on the June 2014 flare on FSRQ 3C 454.3. In section 4 we present our current research using South Africa based telescopes, and we give our final remarks in the conclusions. 


\section{High energy emission in blazars: leptonic versus hadronic}

Theoretical models predict that non-thermal high-energy radiation ( $\mathrm{keV}$ to $\mathrm{TeV}$ domain) can be produced in the jets by both leptonic and hadronic processes (see for example Böttcher et al. [14]). Though in many cases, a simple leptonic scenario - inverse Compton mechanism - reproduces well the data, in some cases hadronic processes seem to reproduce the data more accurately.

\subsection{Broadband SED modelling}

Though the leptonic/hadronic scenarios are often discussed in the context of broadband SED modelling, it is often difficult to be conclusive in ruling out one mechanism over the others. Since two or multiple zone leptonic models, as well as hadronic models, involve many free parameters in the SED fittings, only the one-zone leptonic scenario can easily be ruled out for some cases.

In the case of the inverse Compton mechanism, we distinguish between Synchrotron SelfCompton (SSC) and external Compton (EC) models. In the SSC model, electrons upscatter LE photons produced by the synchrotron mechanism, whereas in the EC model at least one external source of radiation constitutes the seed photons (e.g. from the disk, BLR, torus). Depending on the class of blazar, the SSC/EC ratio varies, since the EC requires the presence of strong external radiation fields such as the ones present around FSRQs. The HE component of the BL Lac SEDs can often be fitted with SSC only. Some major inputs used in the broadband SED fits are - besides the different radiations production mechanisms - the relativistic boosting, the electron energy distribution, the magnetic field, the internal $\gamma \gamma$ absorption, the external extragalactic background light (EBL), etc. (see section 7 in Dermer \& Giebels [7]).

Proper hadronic modelling of the HE component of the SED is more difficult: it demands more intensive computing and requires many more free parameters than in leptonic models. Hadronic emission mechanisms in blazar jets are expected to be mainly initiated by proton synchrotron, which is possible if the protons in the jet are accelerated to $E_{\mathrm{p}}>7 \times 10^{16} E_{p h, e V}^{-1} \mathrm{eV}, E_{p h, e V}$ being the energy threshold of the emitted photons. This would involve extreme values of some parameters, such as a very high magnetic field and a very small gamma-ray emitting region. The proton synchrotron mechanism may also initiate cascades of secondary particles, that include pions, neutrinos, muons and electrons. These secondary electrons can also produce photons through synchrotron radiation. Böttcher [15] gives a well-documented review of the basic features of the leptonic and hadronic modelling of blazars.

Interesting examples of broadband SED modelling can be found in Abdo et al. [16] and Aleksic et al. [17] (leptonic modelling of Mrk 421), Cerruti et al. [18] (hadronic modelling of Mrk 421), and Böttcher et al. [14] (modelling of 12 blazars from different types with both leptonic and hadronic scenarios). In Figures 2 and 3 are shown some of the main results from these papers.

\subsection{Time-domain studies (light-curves)}

We can derive from the above considerations that the variability pattern, as well as the correlation of light-curves between LE and HE components, would bring different signatures that could be 


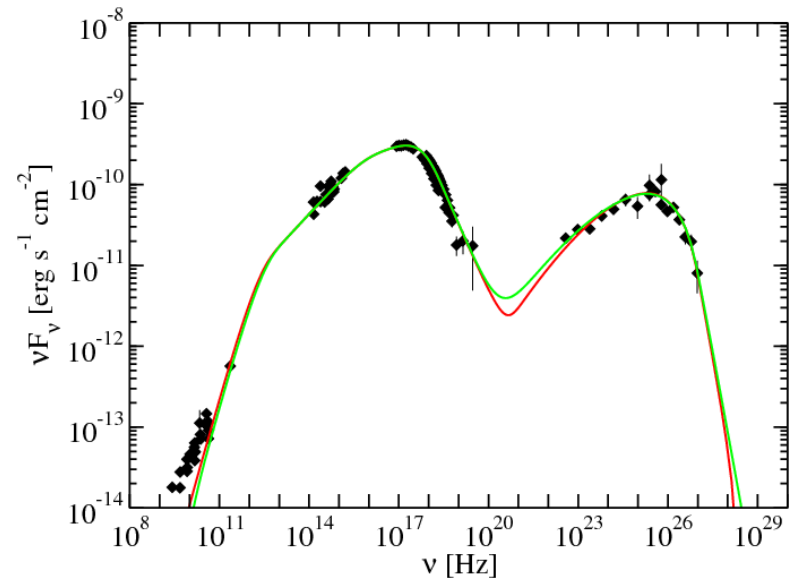

Figure 2: SED of Mrk 421 with two different one-zone SSC model fits obtained with different minimum variability timescales: $t_{v a r}=1$ day (red curve) and $t_{v a r}=1 \mathrm{hr}$ (green curve). Figure 11 from Abdo et al. [16].

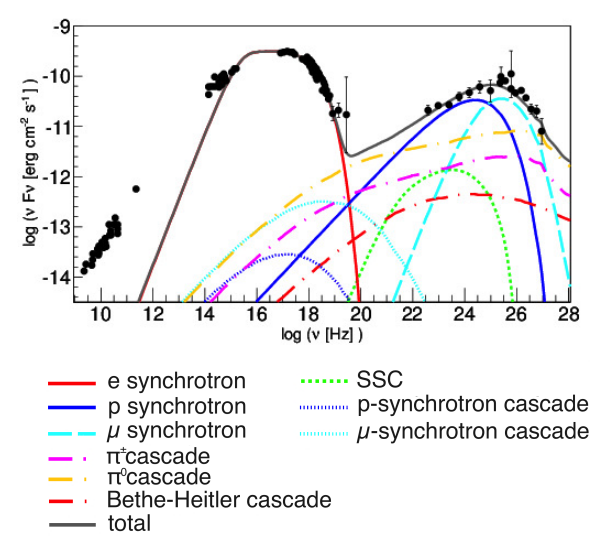

Figure 3: Hadronic modelling of the SED of Mrk 421 (data points taken from Abdo et al. [16]). Data have been corrected for the EBL absorption, which is not included in the model. Figure 1 from Cerruti et al. [18].

used to distinguish between a leptonic and lepto-hadronic modelling of the broadband SED $^{1}$. A fast variability pattern $(\leq 1$ day) of $\mathrm{X}$-ray or gamma-ray light-curves would indicate a leptonic origin of the HE radiation, due to the more rapid cooling time scale of electrons as compared to protons. Also, a high level of correlation between LE and HE light-curves may indicate that the radiation is initiated by the same electron population (leptonic scenario). However, the multiwavelength study of the light-curve patterns for several flaring episodes, though easy to undertake, is not enough to distinguish between leptonic and hadronic scenarios. Indeed, some alternative explanations of the fast and correlated variability have been proposed, such as the modelling of the HE component by a proton-synchrotron scenario with a magnetic field $\mathrm{B} \sim 100 \mathrm{G}$ (e.g. [19]).

\subsection{Polarisation at HE}

Measurement of polarisation at $\mathrm{HE}$ is expected to be a powerful tool for discrimination between leptonic and hadronic models. Unfortunately, the sensitivity of previous X-ray/soft gammaray missions was not enough to detect polarisation from blazars, though INTEGRAL detected polarisation from a small number of bright Galactic sources [20] and gamma-ray bursts (e.g. [21]). Recently launched X-ray missions, such as ASTROSAT, are expected to have a high sensitivity for polarisation measurements. Monte Carlo simulations predict a possible detection of polarisation in the 30-200 MeV range by Fermi-LAT [22]. However, only a small percentage of gamma-ray-like events in the real data could be selected, which would also limit the observation of the polarisation to very bright sources only. A theoretical review and modelling of the polarisation measurements that can be expected for a few blazars are presented in Zhang and Böttcher [23]. Figure 4 shows the case for 3C 279, a bright FSRQ. A non-detection of polarisation by sensitive enough experiments

\footnotetext{
${ }^{1}$ We often called "lepto-hadronic" a broadband SED modelling for which the LE component is modelled by electron synchrotron and the HE component by hadronic mechanisms as described above. However, the phrase "hadronic modelling" is commonly understood as referring to the HE component only, the leptonic origin of the LE component being widely accepted.
} 


\section{$3 \mathrm{C} 279$}

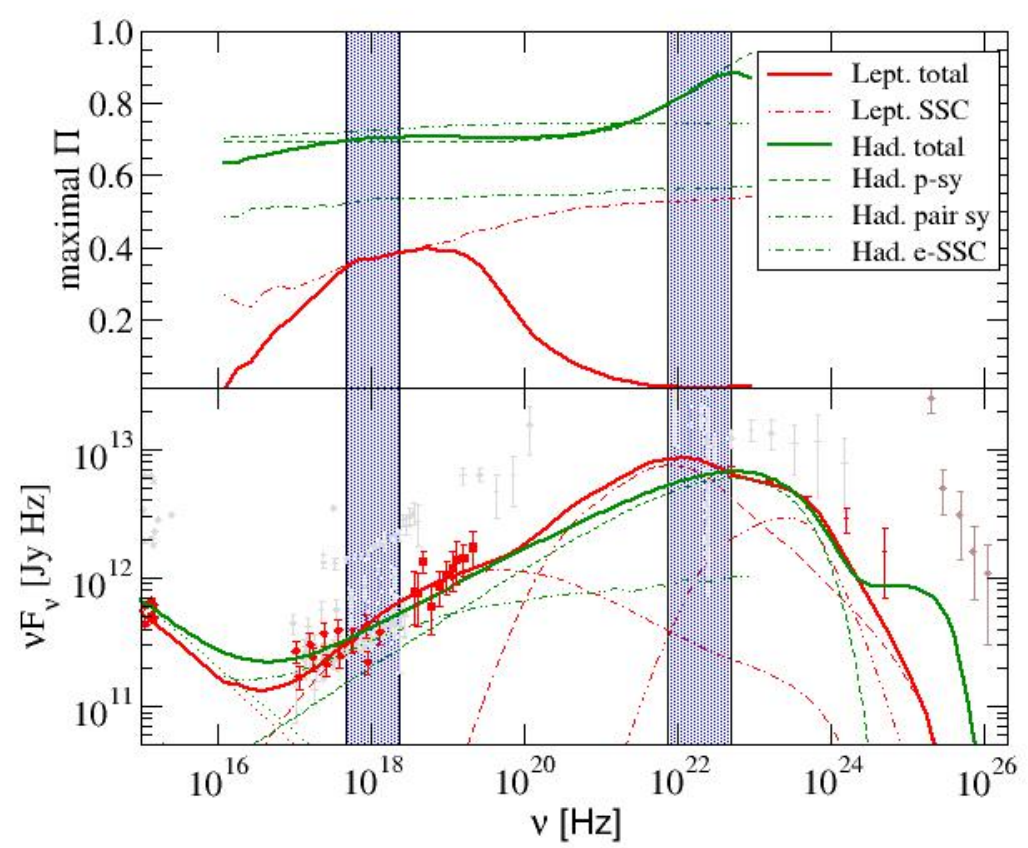

Figure 4: UV through gamma-ray SED (lower panel) and maximum degree of polarisation (upper panel) for the FSRQ 3C279. Leptonic model fits are plotted in red and hadronic models in green. Different lines indicate individual radiation components as labelled in the legend. Shaded areas indicate the 2-10 keV range (X-ray polarimeters), and the $30-200 \mathrm{MeV}$ range (in which gamma-ray emission may be measurable by Fermi-LAT). Figure 2 from Zhang and Böttcher [23].

could indicate either the signature of the unpolarised IC emission or the signature of a polarised hadronic emission in disordered magnetic fields. However, the detection of a certain degree of polarisation would give a strong support to the proton synchrotron mechanism.

\subsection{Association of neutrino events with HE emission from blazars}

The IceCube neutrino observatory has detected more than 52 very-high-energy (VHE) cosmic neutrinos. Due to very large uncertainties on their localisation, it is very difficult to associate them with existing high energy sources (see Figure 5), though some correlation studies were performed (e.g. Moharana et al. [24], Aartsen et al. [25]). A stronger evidence of association would come if a temporal correlation between a neutrino detection and an HE flare from a source is discovered. A hint of such correlation was reported concerning the IceCube event 67093193 detected on 27 April 2016, when PG 1553+113 was reported flaring in the $0.3-10 \mathrm{keV}$ band by Swift-XRT (ATEL \# 8998 and 9008). The IceCube, MAGIC and VERITAS Collaborations have a common follow-up program in such a way that a rapid pointing of at least one of the major Cherenkov telescopes in the Northen Hemisphere will observe the area of the sky from which IceCube would send an alert [26].

In Table 2 are summarised the status and feasibility of studies that can be carried out to provide constraints that may support one type of radiation production mechanism over the other. This shows 


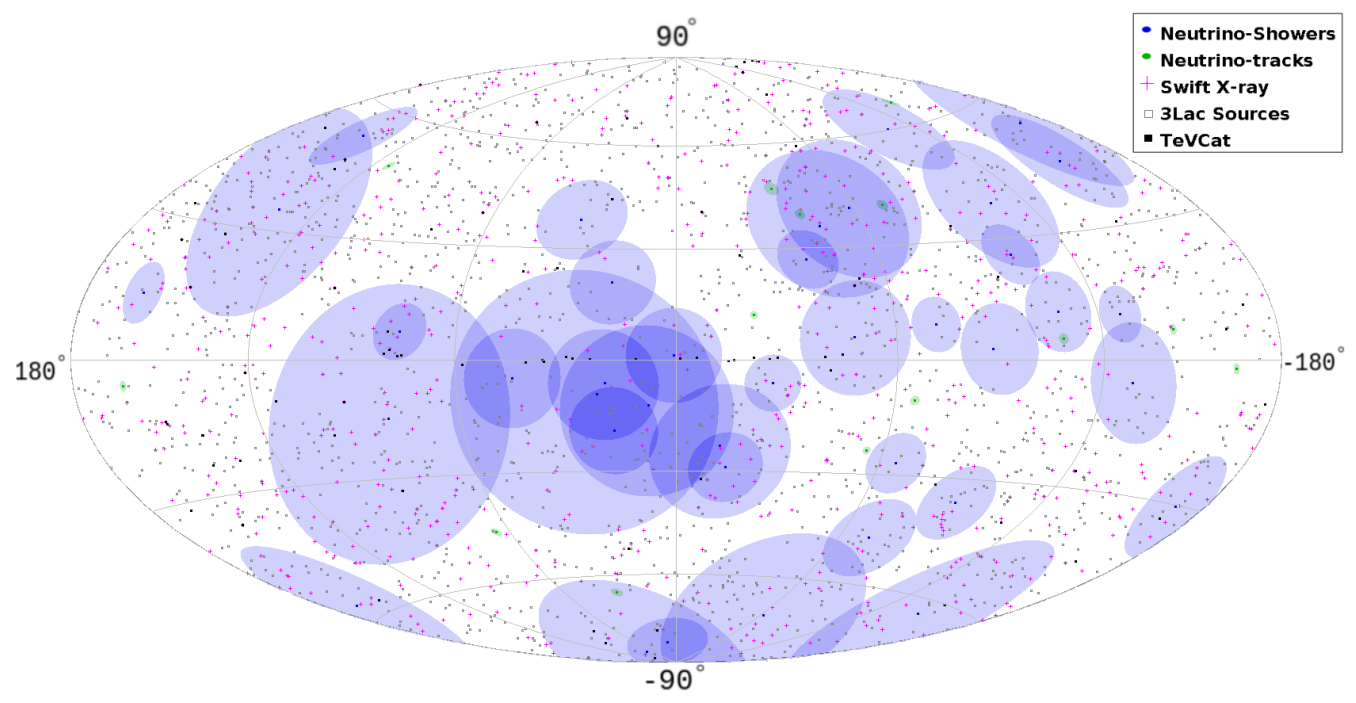

Figure 5: Sky map of the 52 IceCube cosmic neutrino events (as known in 2015) with error circles and sources from different catalogues in the Galactic coordinate system. Figure 1 from Moharana et al. [24].

Table 2: Current methods for investigating the leptonic/hadronic discrepancy in modelling the blazar SEDs.

\begin{tabular}{c|c}
\hline method & feasibility/caveat \\
\hline \hline \multicolumn{2}{c|}{ Light-curves: } \\
\hline $\begin{array}{c}\text { Correlation or non-correlation of } \\
\text { radio (or optical) versus gamma rays }\end{array}$ & easy to analyse - lot of data \\
\hline Fast variability & easy to analyse - lot of data \\
\hline \multicolumn{2}{c}{ Broadband SED: } \\
\hline Fitting of the HE hump & $\begin{array}{c}\text { often too many fit parameters } \\
\text { not always simultaneity of data }\end{array}$ \\
\hline Polarisation in the X-ray and gamma-ray domains & $\begin{array}{c}\text { expected from the newly } \\
\text { launched/future X-ray missions }\end{array}$ \\
\hline
\end{tabular}

Neutrinos detections:

\begin{tabular}{l|l} 
IceCube/X- and gamma-ray correlations & narrow spatial/time coincidences needed
\end{tabular}

the need for more precise multiwavelength observations of blazars, in order to constrain parameters such as the bulk jet Lorentz factor $\Gamma_{\text {jet }}$, the magnetic field, HE polarisation.

\section{Fast variability of FSRQ 3C 454.3 during June 2014}

Note. The results and the discussion presented in this section are taken from a previous work published by R. J. Britto, E. Bottacini, B. Lott, S. Razzaque and S. Buson [27]. 


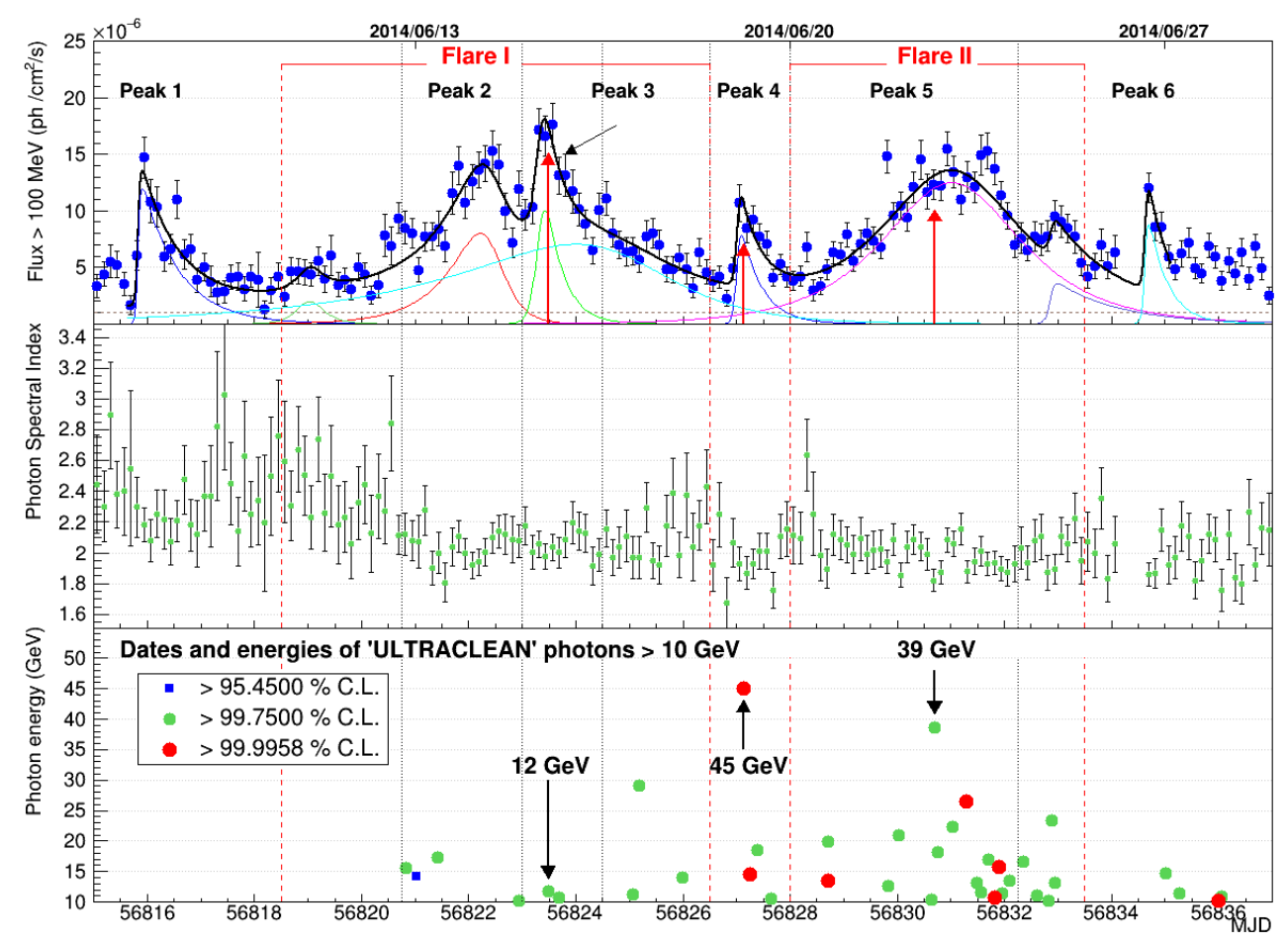

Figure 6: Top panel: Fermi-LAT light-curve of the flare phase with 3-hr binning. Flaring peaks were fitted by the $F$ function (in Equation 3.1) for nine structures. Fits were performed in the MJD 56815.62556835.130 time range. The thin colour lines correspond to the contribution of single peaks in the total fit, which is represented by the thick black line. The dashed brown line is the fitted constant baseline, which also contributes to the total fit. Six major peaks were labelled for more detailed studies. The red arrows indicate the arrival time of the three high-energy photons used to calculate the Doppler factor, and whose energies are labelled in the bottom panel. Due to an instrumental problem, a few bins around MJD 56834 contains no data. Middle panel: Photon spectral index $(\Gamma)$ of the PL fits of data. Bottom panel: Arrival time and energy of $\mathrm{E}>10 \mathrm{GeV}$ photons with three different significance levels of source association (2-, 3-, and 4- $\sigma$ Gaussian equivalent). Vertical red dash lines indicate the two major flaring phases (I and II), and black dotted lines indicate Peaks 2, 3, 4 and 5. Figure 2 of [27].

As mentioned in section 1, Fermi-LAT is a particularly well-suited instrument for the monitoring of flaring episodes of blazars. Whenever a specific outburst is reported, time-domain analysis is performed on a specific time interval. A fast variability may provide a constraint on the size of the gamma-ray emitting region. Also, correlation studies of light-curves between different wavelength ranges are useful to discuss similarities of physical processes that may be common in different frequency domains.

We (Britto et al. [27]) undertook the study of the fast variability pattern of 3C 454.3 during its 7-29 June 2014 flare, using Fermi-LAT data between $100 \mathrm{MeV}$ and $300 \mathrm{GeV}$. A standard unbinned likelihood analysis was performed, using the Pass 8 data and analysis package. Details on the analysis procedure are given in Britto et al. [27]. Light-curves were produced by using a single power law spectral (PL) model of our source of interest. 
Through a detailed observation of a 3-hr light-curve (Figure 6) we could identify a few individual flaring structures that we fitted using the following function:

$$
F(t)=2 F_{0}\left(e^{\left(t_{0}-t\right) / T_{r}}+e^{\left(t-t_{0}\right) / T_{f}}\right)^{-1},
$$

where:

- $t_{0}$ is the time of the peak value;

- $T_{r}$ and $T_{f}$ are the rise and fall time, respectively;

- $F_{0}$ is the flux at $t_{0}$, representing the amplitude of the subflare.

A time-domain unbinned method was also used to more precisely estimate the rise-time $T_{r}$ of two of the sharpest peaks, labelled as "Peak 1" (at MJD 56816) and "Peak 4" (at MJD 56827). For this study, we used a function of the form $S_{S}(E, t)=S(E) \times(F(t)+B)$, where $F(t)$ is given in Equation 3.1, $B$ is a constant component and $S(E)$ is a power-law distribution. We show in Figure 7 different features concerning the ("Peak 4") flare. The top panel displays the LAT exposure rate for $3 \mathrm{C} 454.3$. The bottom panel shows the 3-hr light-curves as well as the function fitted on the 3-hr points (blue) and the function resulting from the fit by the unbinned method (red). To enable a verification of the fit quality, a comparison between the counts of photons ascribed to the source between the data (blue) and the estimate from the fitted function (red) is given in the middle panel. The unbinned method gives a rise-time $T_{r}=1.2 \pm 0.7 \mathrm{ks}(0.3 \pm 0.2 \mathrm{hr})$ in good agreement with the results of the conventional fit, but with better-defined uncertainties. $T_{r}$ in Peak 4 is the shortest timescale reported for this source in the gamma-ray band.

This study, which we carried out on the June 2014 flare of 3C 454.3, is a good example of probing gamma-ray absorption processes, and providing limits on the jet parameters such as the Doppler factor $\delta$, the Lorentz factor $\Gamma_{\text {jet }}$, and the size and distance of the blob to the SMBH, though it requires some generic assumptions, such as a spherical blob diameter to equal the cross-section of the jet (Figure 1). However, a fast variability of the source places a more direct constraint on the size of the emitting region, by considering the light path difference between different regions of the blob. A variability pattern such as the one we discussed here is expected to be due to gamma-ray emission from blobs located within the jet. In this case, the radius of the blob can be expressed as $R^{\prime} \approx \delta c t_{v} /(1+z)$.

The value of $\delta$ can be constrained by requiring the blob to be optically thin to $\mathrm{GeV}$ photons that may be absorbed through the $\gamma \gamma \rightarrow e^{ \pm}$pair production process. For each peak (associated to a single blob), gamma-rays of energy $E_{\gamma}$ from 3C 454.3 should be interacting with an HE radiation field (mainly dominated by $\mathrm{keV}$ photons). We modelled this $\mathrm{HE}$ radiation field by fitting the quasisimultaneaous Swift-XRT and Fermi-LAT SEDs, assuming that the X-ray photons are produced in the same blob as the gamma rays. Requiring the $\gamma \gamma$ opacity for the $45 \mathrm{GeV}$ photon emitted during "Peak 4" to be less or equal to one, we calculated a lower limit on $\delta \sim 26-29$.

Observations from long-term Very Long Baseline Interferometry (VLBI) inferred an average value of the angle between the jet and our line-of-sight to be $\theta=1.3^{\circ}$ [28]. Using $\delta=\left[\Gamma_{\text {jet }}(1-\right.$ $\left.\left.\beta_{\text {jet }} \cos \theta\right)\right]^{-1}$, we calculated lower limits on the Lorentz factor $\Gamma_{\text {jet }} \gtrsim 15$ for Peak 4 . This value is compatible with the ones reported by Jorstad et al. [28] and Sikora et al. [29] $\left(\Gamma_{\text {jet }} \sim 20\right)$. 


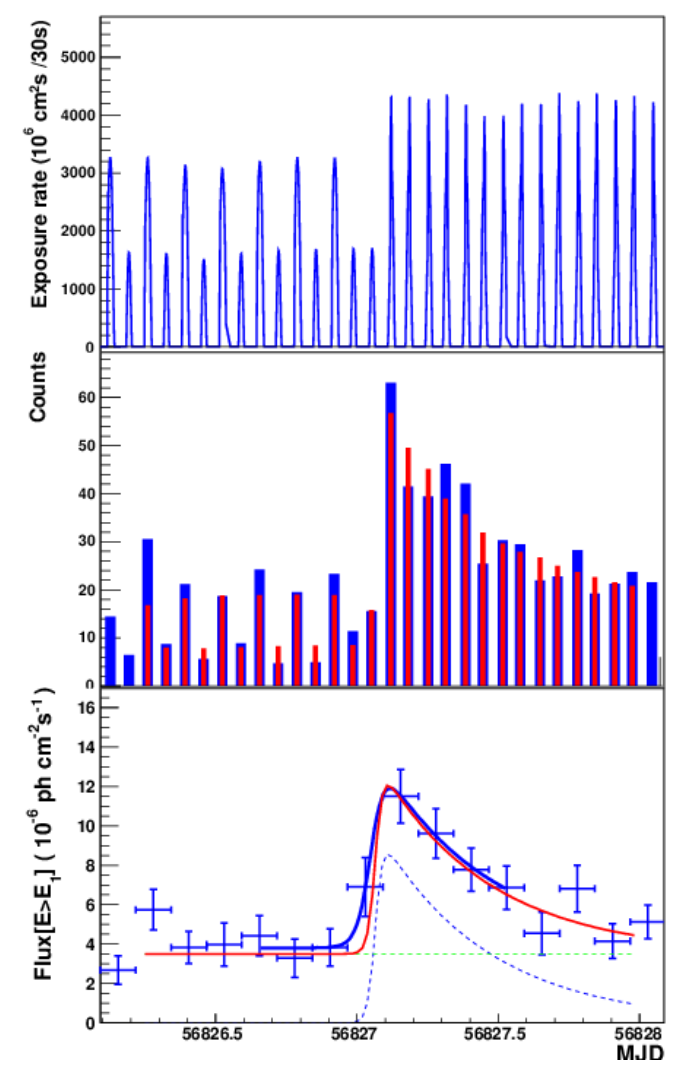

Figure 7: MJD 56827 ("Peak 4") flare. Top panel: Exposure rate as a function of time. Middle panel: Measured (probability-weighted, blue) and modelled (red) photon counts. Bottom panel: light-curves. Blue points: 3-hr binning. Blue curve: function fitted to 3-hr points. Red curve: function fitted using the unbinned method. The green dashed line corresponds to the constant component and the blue one to the flare component $F(t)$ given in Equation 3.1. Figure 4 of Britto et al. [27].

We then derived the radius of the blob to be $R^{\prime} \gtrsim 5.6 \times 10^{14} \mathrm{~cm}$ for Peak 4 , and $R^{\prime} \gtrsim 2.3 \times 10^{16}$ $\mathrm{cm}$ for Peak 5. We also derive, by using the relation $r \simeq 2 \Gamma_{\text {jet }}^{2} c t_{v} /(1+z)$, the distances $r$ from the SMBH $r \gtrsim 1.0 \times 10^{16} \mathrm{~cm}$ for Peak 4 , and $r \gtrsim 1.8 \times 10^{17} \mathrm{~cm}$ for Peak 5. This would locate these blobs in the outer layers of the canonical BLR, estimated to be $R_{B L R}<10^{18} \mathrm{~cm}$ for $3 \mathrm{C} 454.3$ $[30,31]$.

\section{Observations of Fermi-LAT blazars using Southern Africa based telescopes}

Southern Africa based telescopes offer a good potential for photometric and spectroscopic observations of optical counterparts of Fermi-LAT blazars. We used the SpUpNIC spectrograph on the 1.9-m telescope and the Robert Stobie Spectrograph (RSS) on the Southern African Large Telescope (SALT-RSS, see Figure 8), both located at the South African Astronomical Observatory (SAAO [20 48' 42" E ; - 32 23' 14" S; altitude:1798 m]), Sutherland, South Africa. Our main interest is the observation of blazar candidates of uncertain type (BCUs) from the Fermi-LAT Third 


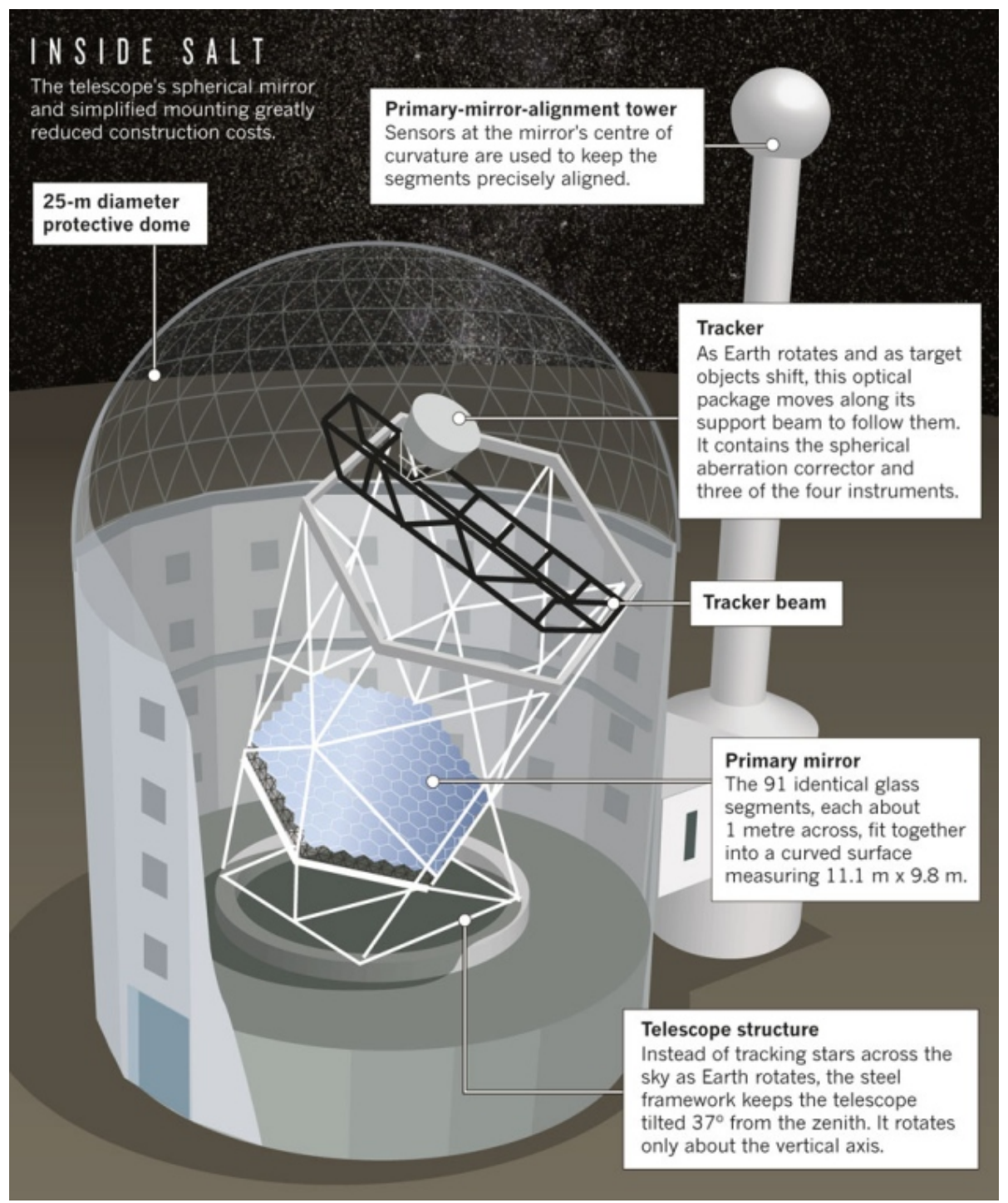

Figure 8: Layout of the Southern African Large Telescope (SALT), located near the small town of Sutherland, in the Northern Cape province, South Africa, about $400 \mathrm{~km}$ from Cape Town. In full science operation since 2011, SALT is funded by a consortium of international partners from South Africa, the United States, Germany, Poland, India, the United Kingdom and New Zealand. Figure from Nordling [32].

AGN Catalog (3LAC), as presented by B. van Soelen in this conference [33], and previously reported by Klindt et al. [34, 35]. Our first BCU to be classified by combining observations with telescopes based in South Africa is NVSS J141922-083830. Following an alert from MASTER ${ }^{2}$ Kislovodsk on 21 February 2015 [38], a spectrum was obtained with SALT-RSS on 1 March 2015, and its redshift was identified to be $\mathrm{z}=0.903$ if the detected emission line is interpreted to be the [MgII 2798] Å line [39, 40].

Besides the classification of BCUs, we also study other well-known flaring blazars [36, 37].

\footnotetext{
${ }^{2}$ Acronym for Mobile Astronomical System of TElescope-Robots
} 
Also, the newly commissioned polarimetry capability of SALT-RSS allowed us to measure both the degree of polarisation and the polarisation angle as a function of the wavelength. This work on spectropolarimetry is performed in collaboration with the group lead by M. Böttcher at North-West University, Potchefstroom, South Africa.

From all 3LAC sources that are variable, belonging to the BL Lac or FSRQ or BCU classes, and observable by the SALT telescope in South Africa $\left(-75^{\circ} \lesssim\right.$ declination $\left.\lesssim+10^{\circ}\right)$, we have a list of $\approx 140$ target sources with a magnitude $\mathrm{V}$ brighter than 19 . Besides the telescopes we use for spectroscopy, several optical robotic telescopes perform photometric monitoring and observation, such as MASTER-SAAO (Russia-South Africa Collaboration, PI V. Lipunov) at Sutherland, and Watcher (Ireland, PI: L. Hanlon) at the Boyden Observatory, Bloemfontein. We also use the Boyden $1.5 \mathrm{~m}$ telescope for photometry.

We acknowledge our collaboration with S. Chandra from TIFR, Mumbai, as part of the SALTblazar team, and our collaboration with G. Chiaro et al. [41], in their work on classifying BCUs using a statistical method based on artificial neural network.

\section{Conclusions}

The objective of this paper was to illustrate how the investigation of the variability and broadband spectral features of blazars can provide constraints on the physical properties of the radiation emission mechanisms. We focused on the study of FSRQ 3C 454.3 during the June 2014 flare, and provided a constraint on the size and location of the gamma-ray emitting blob, using fast variability features and opacity to the HE radiation. In our final section, we briefly described our work and prospects of using South Africa based telescopes. The SALT telescope is a particularly well-suited instrument to monitor many faint blazars (magnitude $\mathrm{V} \lesssim 19$ ) in order to determine redshifts and provide classification of BCUs. This contributes to the effort of collecting more multiwavelength data with sensitive instruments in order to improve our current understanding of the physics of AGNs and their evolution.

\section{Acknowledgements}

The Fermi-LAT Collaboration acknowledges support for LAT development, operation and data analysis from NASA and DOE (United States), CEA/Irfu and IN2P3/CNRS (France), ASI and INFN (Italy), MEXT, KEK, and JAXA (Japan), and the K.A. Wallenberg Foundation, the Swedish Research Council and the National Space Board (Sweden). Science analysis support in the operations phase from INAF (Italy) and CNES (France) is also gratefully acknowledged. The authors acknowledge support from the National Research Foundation, South Africa and the South African Gamma-ray Astronomy Programme (SA-GAMMA). We thank the anonymous referee for the review of our paper and for his/her valuable comments.

Note. A paper with a similar material was presented at the Annual Conference of the French Society of Astronomy and Astrophysics (SF2A 2016). However, the conference proceeding of the SF2A conference was written in a complementary way to this one. The reader may then be interested to refer to Britto et al. [42]. 


\section{References}

[1] M. Schmidt, 3C 273: A Star-Like Object with Large Red-Shift, Nature, 197 (1963), 1040.

[2] C. Hazard, M. B. Mackey \& A. J. Shimmins, Investigation of the Radio Source 3C273 by the method of Lunar Occultations, Nature, 197 (1963), 1037

[3] Thomas A. Matthews \& Allan R. Sandage, Optical Identification of 3C 48, 3C 196, and 3C 286 with Stellar Objects, ApJ, 138 (1963), pp. 30-56

[4] J. L. Greenstein \& Thomas A. Matthews, Red-Shift of the Unusual Radio Source 3C48, Nature, 197 (1963), pp. 1041-1042

[5] C. M. Urry \& P. Padovani, Unified Schemes for Radio-Loud Active Galactic Nuclei, Publications of the Astronomical Society of the Pacific, 107 (1995), 803

[6] F. Massaro, D. J. Thompson \& E. C. Ferrara, The Extragalactic Gamma-ray Sky in the Fermi era, Astron. Astrophys. Rev., 24 (2016), 2.

[7] C. D. Dermer \& B. Giebels, Active galactic nuclei at gamma-ray energies, Article in Comptes Rendus de Physique, 17 (6), February 2016, arXiv:1602.06592 [astro-ph.HE]

[8] J. D. Finke, Modeling Fermi Large Area Telescope and Multiwavelength Data from Blazars, Proceedings of Science, POS (HEASA2 015 ) (2015) 006, 21pp, arXiv:1602.05965 [astro-ph.HE], Proceedings of the 3rd Annual Conference on High Energy Astrophysics in Southern Africa (HEASA2015), 18-20 June 2015, Johannesburg, South Africa, Eds. M. Böttcher, D. Buckley, S. Colafrancesco, P. Meintjes and S. Razzaque

[9] D. J. Saikia \& C. J. Salter, Polarization properties of extragalactic radio sources, Ann. Rev. Astron. Astrophys., 26 (1988), 93

[10] S. Trippe, R. Neri, M. Krips et al., The first IRAM/PdBI polarimetric millimeter survey of active galactic nuclei. I. Global properties of the sample, A\&A, 515 (2010), A40

[11] S. Trippe, R. Neri, M. Krips et al., The first IRAM/PdBI polarimetric millimeter survey of active galactic nuclei. II. Activity and properties of individual sources, A\&A, 540 (2012), A74

[12] I. Agudo, C. Thum, H. Wiesemeyer \& T. P. Krichbaum, A 3.5mm polarimetric survey of radio-loud active galactic nuclei, ApJS, 189 (2010), 1

[13] W. B. Atwood, A. A. Abdo, M. Ackermann et al., The Large Area Telescope on the Fermi Gamma-Ray Space Telescope Mission, ApJ, 697 (2009), pp. 1071-1102

[14] M. Böttcher, A. Reimer, K. Sweeney, A. Prakash, Leptonic and Hadronic Modeling of Fermi-Detected Blazars, ApJ, 768 (2013), 54, 14pp.

[15] M. Böttcher, in Proc. Fermi meets Jansky - AGN at Radio and Gamma-Rays, MPIfR, Bonn, Germany, June 21-23, 2010, Savolainen, T., Ros, E., Porcas, R.W., \& Zensus, J.A. (eds.), Models for the Spectral Energy Distributions and Variability of Blazars

[16] A. A. Abdo, M. Ackermann, M. Ajello et al, Fermi Large Area Telescope Observations of Markarian 421: The Missing Piece of its Spectral Energy Distribution, ApJ, 736 (2011), 131, 22pp.

[17] J. Aleksić, S. Ansoldi, L. A. Antonelli et al, Unprecedented study of the broadband emission of Mrk 421 during flaring activity in March 2010, A\&A, 578 (2015), A22

[18] M. Cerruti, A. Zech, C. Boisson and S. Inoue, A hadronic origin for ultra-high-frequency-peaked BL Lac objects, MNRAS, 448 (2015), pp. 910-927 
[19] F. A. Aharonian, TeV gamma rays from BL Lac objects due to synchrotron radiation of extremely high energy protons, New Astron., 5 (2000), pp. 377-395, arXiv:astro-ph/0003159

[20] P. Laurent, D. Götzb, C. Gouiffès, Integral observations of polarization: from the Crab pulsar to Cygnus X-1, Proceedings of Science, POS (INTEGRAL 2012) (2012) 005, 8pp, arXiv:1408.4121 [astro-ph.HE], Proceedings of "An INTEGRAL view of the high-energy sky (the first 10 years)" - 9th INTEGRAL Workshop and celebration of the 10th anniversary of the launch (INTEGRAL 2012). 15-19 October 2012. Bibliotheque Nationale de France, Paris, France

[21] D. Götz, P. Laurent S. Antier et al., GRB 140206A: the most distant polarized gamma-ray burst, MNRAS, 444 (2014), pp. 2776-2782

[22] Matteo Giomi, Rolf Bühler, Carmelo Sgrò, Francesco Longo \& W. B. Atwood, Estimate Of The Fermi Large Area Telescope Sensitivity For Gamma-ray Polarization, AIP Conference Proceedings, 1792 (2017), 070022; doi: http://dx.doi.org/10.1063/1.4969019

[23] H. Zhang and M. Böttcher, X-Ray and Gamma-Ray Polarization in Leptonic and Hadronic Jet Models of Blazars, ApJ, 774 (2013), 18 (7pp)

[24] R. Moharana, R. J. Britto and S. Razzaque, Search for extragalactic astrophysical counterparts of IceCube neutrino events, Proceedings of ICRC 2015 (2016), arXiv:1602.03694

[25] M. G. Aartsen, K. Abraham, M. Ackermann et al., All-sky search for time-integrated neutrino emission from astrophysical sources with 7 years of IceCube data, ApJ, 835 (2017), 151A, arXiv:1609.04981 [astro-ph.HE]

[26] M. G. Aartsen, K. Abraham, M. Ackermann et al., Very High-Energy Gamma-Ray Follow-Up Program Using Neutrino Triggers from IceCube, Journal of Instrumentation, 11 (2016), P11009, arXiv:1610.01814 [hep-ex]

[27] R. J. Britto, E. Bottacini, B. Lott, S. Razzaque \& S. Buson, Fermi-LAT Observations of the 2014 May-July Outburst from 3C 454.3, ApJ, 830 (2016), 162 (15 pp), arXiv:1511.02280 [astro-ph.HE]

[28] S. G. Jorstad, A. P. Marscher, M. L. Lister et al., Polarimetric Observations of 15 Active Galactic Nuclei at High Frequencies: Jet Kinematics from Bimonthly Monitoring with the Very Long Baseline Array, AJ, 130 (2005), pp. 1418-1465

[29] M. Sikora, R. Moderski, and G. M. Madejski, 3 C 454.3 Reveals the Structure and Physics of Its “Blazar Zone”, ApJ, 675 (2008), pp. 71-78

[30] G. Bonnoli, G. Ghisellini, L. Foschini, F. Tavecchio and G. Ghirlanda, The $\gamma$-ray brightest days of the blazar 3C 454.3, MNRAS, 410 (2011), pp. 368-380

[31] T. Sbarrato, G. Ghisellini, L. Maraschi and M. Colpi, The relation between broad lines and $\gamma$-ray luminosities in Fermi blazars, MNRAS, 421 (2012), pp. 1764-1778

[32] L. Nordling, Astronomy: Southern star - Can the Southern African Large Telescope live up to its potential?, Nature, $\mathbf{5 0 2}$ (2013), pp. 608-610

[33] B. van Soelen, L. Klindt, J. P. Marais et al, New Discoveries and Surprises Revealed through SALT Spectroscopy of the Unclassified Fermi-LAT Sources, in these proceedings

[34] L. Klindt, B. van Soelen, P. J. Meintjes and P. Väisänen, Optical spectroscopic observations of unclassified Active Galactic Nuclei in the Fermi-2LAC catalogue, Proceedings of SAIP2015: the 60th Annual Conference of the South African Institute of Physics (2016) 
[35] L. Klindt, B. van Soelen, P. J. Meintjes and P. Väisänen, Optical spectroscopic classification of a selection of Southern hemisphere Fermi-LAT unclassified blazars, MNRAS, 467 (2017), pp. $2537-2544$

[36] R. J. Britto, Study of flaring quasars using optical/gamma-ray correlations, Proceedings of Science, SALT Science Conference 2015, PoS (S SC2015) (2015) 032, STIAS, Stellenbosch, South Africa, $1-5$ June 2015

[37] R. J. Britto, J. P. Marais, P. J. Meintjes et al., Observations of the flaring Fermi-LAT blazar 4C +01.02 and prospects in spectro-polarimetry with SALT-RSS, Proceedings of Science, POS (HEASA2016) (2015) 021, Proceedings of the 4th Annual Conference on High Energy Astrophysics in Southern Africa (HEASA 2016), 25-26 August 2016, SAAO, Cape Town, South Africa, Eds. M. Boettcher, D. Buckley, S. Colafrancesco, P. Meintjes and S. Razzaque

[38] V. Lipunov, E. Gorbovskoy, V. Kornilov et al., MASTER: very bright blazar flare, ATEL 7133 (2015)

[39] D. A. H. Buckley, J. B. Breytenbach, A. Kniazev et al., SALT spectroscopy of the flaring blazar J141922.55-083832.0, ATEL 7167 (2015)

[40] D. A. H. Buckley et al., in preparation

[41] G. Chiaro, D. Salvetti, G. La Mura et al., Blazar Flaring Patterns (B-FlaP): Classifying Blazar Candidates of Uncertain type in the third Fermi-LAT catalog by Artificial Neural Networks, MNRAS, 462 (2016), pp. 3180-3195

[42] R. J. Britto, E. Bottacini, M. Böttcher et al., Multiwavelength Study of Fermi-LAT blazars Variability and Radiation Production Mechanisms, SF2A-2016: Proceedings of the Annual meeting of the French Society of Astronomy and Astrophysics. Eds.: C. Reylé, J. Richard, L. Cambrésy, M. Deleuil, E. Pécontal, L. Tresse and I. Vauglin, held 14-17 June, 2016 at the Centre de Recherche Astrophysique de Lyon, pp. 93-101 